\title{
Ganglioside GM3 exerts opposite effects on motility via epidermal growth factor receptor and hepatocyte growth factor receptor-mediated migration signaling
}

\author{
YING LI ${ }^{1}$, XIAOHUA HUANG ${ }^{2,3}$, CONGCONG WANG $^{2}$, YUZHONG LI $^{1}$, MINGCHUN LUAN $^{4}$ and KELI MA ${ }^{2}$ \\ ${ }^{1}$ Department of Clinical Laboratory, Second Affiliated Hospital of Dalian Medical University, Dalian, Liaoning 116023; \\ ${ }^{2}$ Department of Biochemistry and Molecular Biology, Dalian Medical University; ${ }^{3}$ Department of Clinical Biochemistry, \\ College of Laboratory Medicine, Dalian Medical University, Dalian, Liaoning 116044; ${ }^{4}$ Department of \\ Microbiology Laboratory, Dalian Center for Disease and Prevention, Dalian, Liaoning 116021, P.R. China
}

Received January 2, 2014; Accepted October 24, 2014

DOI: $10.3892 / \mathrm{mmr} .2014 .3087$

\begin{abstract}
The ganglioside GM3 exerts its different effects via various growth factor receptors. The present study investigated and comparatively analyzed the opposing effects exerted by GM3 on the migration of mouse hepatocellular carcinoma Hepa1-6 cells via epidermal growth factor receptor (EGFR) and hepatocyte growth factor receptor (HGFR/cMet). The results demonstrated that GM3 inhibited EGF-stimulated motility, but promoted HGF-stimulated motility of the Hepa1-6 cells via phosphatidylinositol 3-kinase/Akt-mediated migration signaling. It is well established that the main cytokines modulating cell proliferation, invasion and metastasis are different in different types of tumor. This difference may, at least in part, explain why GM3 exerted its actions in a tumor-type specific manner.
\end{abstract}

\section{Introduction}

Gangliosides are sialic acid-containing glycosphingolipids, which are generally localized on the outer leaflet of the plasma membrane due to the insertion of their hydrophobic ceramide portion into the membrane, with their oligosaccharide moiety being exposed on the cell surface. Therefore, gangliosides are able to interact with other components of the membrane, including receptors, cellular antigens, adhesion molecules and the extracellular matrix (1). Additionally, gangliosides are involved in the regulation of various processes, including cell proliferation and differentiation, apoptosis, embryogenesis and oncogenesis (2). Each cell type exhibits a distinct pattern of

Correspondence to: Professor Keli Ma, Department of Biochemistry and Molecular Biology, Dalian Medical University, 9 West Lushun South Road, Dalian, Liaoning 116044, P.R. China E-mail: makelipaper@163.com

Key words: Akt, epidermal growth factor receptor, GM3, hepatocyte growth factor receptor, metastasis ganglioside content, which is altered by neoplasia (3-5) and tumor cells or oncogene-transformed cells exhibit a different ganglioside composition compared with non-transformed cells. Among the gangliosides, GM3 is the simplest member and is a common precursor for the more complex gangliosides (6). The involvement of GM3 in the malignant activity of tumor cells, including cell proliferation, adhesion, invasion and metastasis, has been an area of particular interest (7-10). However, the precise mechanism remains to be elucidated. GM3 exerts its actions in a tumor-type specific manner and exerts either inhibitory actions or enhancing actions on tumor growth, migration and metastasis in different types of tumor. It also suppresses the proliferative and invasive potential of various types of cell (11-15). However, an inverse correlation is observed between the proliferative and metastatic properties of cells and their GM3 content in certain types of tumor $(16,17)$. This indicates that GM3 may exert different or opposing actions in different types of tumor and, until recently, the underlying mechanism of GM3 cell-type specificity remained to be elucidated.

To elucidate the underlying mechanism by which GM3 exerted these opposing actions on Hepa1-6 cell migration, the effects of GM3 on the tyrosine residue phosphorylation of EGF receptor (EGFR) and HGF receptor (HGFR) were examined and the activity of certain signaling pathways, which are essential for the modulation of tumor cell growth, invasion, migration and metastasis were investigated.

\section{Materials and methods}

Antibodies. The following antibodies were used: Anti-phosphorylated(p)-EGFR (Tyr-1173) immunoglobulin rabbit polyclonal IgG, anti-p-EGFR (Tyr-845) rabbit polyclonal IgG, anti-p-EGFR (Tyr-1086) rabbit polyclonal IgG, anti-p-EGFR (Tyr-1068) rabbit polyclonal IgG, anti-p-HFGR (Met; Tyr1313) rabbit polyclonal IgG, ant-p-Met (Tyr-1349) rabbit polyclonal IgG, ant-p-Met (Tyr-1365) rabbit polyclonal $\mathrm{IgG}$, anti-Akt1/2/3 rabbit polyclonal $\mathrm{IgG}$, anti-P-Akt1/2/3 (Ser473) rabbit polyclonal IgG, anti-P-Akt1/2/3 (Thr308) rabbit polyclonal IgG, anti-phospholipaseC $\gamma(\mathrm{PLC} \gamma) 1$ rabbit 
polyclonal IgG, anti-p-PLC $\gamma 1$ (Tyr-783) rabbit polyclonal $\mathrm{IgG}$, anti-p-p44/42 mitogen-activated protein kinase (MAPK) rabbit polyclonal IgG (Erk1/2; Thr-202/Tyr-204), anti- $\beta$-actin mouse polyclonal IgG, goat anti-rabbit polyclonal horseradish peroxidase-conjugated IgG, rabbit anti-goat polyclonal horseradish peroxidase-conjugated IgG, goat anti-mouse polyclonal horseradish peroxidase-conjugated IgG and horseradish peroxidase-labeled streptavidin (all from Santa Cruz Biotechnology, Inc., Santa Cruz, CA, USA). Anti-GM3 IgM was obtained from Wako Pure Chemical Industries Ltd (Osaka, Japan) The dilution used was 1:500 for all antibodies.

Reagents. LY294002 was obtained from Sigma-Aldrich (St. Louis, MO, USA), EGF was obtained from ProSpec-Tany TechnoGene, Ltd. (East Brunswick, NJ, USA), Lipofectamine $^{\text {TM }} 2000$ and opti-MEM ${ }^{\text {TM }}$ were obtained from Invitrogen Life Technologies, Carlsbad, CA, USA, Protease inhibitor cocktail was obtained from Sigma-Aldrich and GM3 and GM2 were purchased from Sigma-Aldrich.

Cells and cell culture. Mouse hepatocellular carcinoma Hepa1-6 cells were grown in $10-\mathrm{cm}$ cell culture dishes or in multi-well plates in RPMI-1640 medium supplemented with $10 \%$ heat-inactivated fetal bovine serum (FBS), $100 \mathrm{U} / \mathrm{ml}$ penicillin, and $100 \mathrm{mg} / \mathrm{ml}$ streptomycin at $37^{\circ} \mathrm{C}$ in a $5 \% \mathrm{CO}_{2}$ atmosphere for $48 \mathrm{~h}$.

RNAi GM3 synthase plasmid construction and selection. A negative control and four types of GM3 synthase short hairpin (sh)RNA plasmids were constructed, as follows: PGPU6/green fluorescent protein (GFP)/Neo-shNC (negative control), PGPU6/GFP/Neo-St3ga15-mus-469, PGPU6/GFP/Neo-St3 ga15-mus-613, PGPU6/GFP/Neo-St3ga15-mus-772 and PGPU6/GFP/Neo-St3ga15-mus-832. To select an effective shRNA plasmid that inhibited GM3 synthase, the Hepa1-6 cells were transfected with the GM3 synthase shRNA plasmids using Lipofectamine ${ }^{\mathrm{TM}} 2000$ in opti-MEM ${ }^{\mathrm{TM}}$. The ratio of DNA $(\mu \mathrm{g})$ to lipofectamine $2000(\mu \mathrm{l})$ was 1:3. The effective GM3 synthase shRNA plasmid was selected using reverse transcription polymerase chain reaction (RT-PCR).

Total RNA was isolated from cells using Trizol (Invitrogen Life Technologies), and RT-PCR was conducted with Taq polymerase, according to the manufacturer's instructions (Boehringer-Mannheim, Indianapolis, IN, USA). The cycling parameters were implemented as follows: denaturation at $94^{\circ} \mathrm{C}$ for $30 \mathrm{sec}$, annealing at $58-61^{\circ} \mathrm{C}$ for $1 \mathrm{~min}$ (temperature dependant on primer specifications), and elongation at $72^{\circ} \mathrm{C}$ for $1 \mathrm{~min}$. The number of cycles varied between 25 and 35, depending on the quantity of mRNA. The identity of the PCR products was confirmed through sequencing. The relative quantitative analysis was normalized to that of the endogenous reference gene, glyceraldehyde-3-phosphate dehydrogenase (GAPDH). The mouse GM3 synthase forward primer used was 5'-AGC TGCCAGAGGTGATGAGT-3' and its reverse primer was 5'-TCAAGTGGCTTCAAGCAATG-3'. The mouse GAPDH forward primer used was 5'-TACTTATGCCGATGTCGT TGT-3' and its reverse primer was 5'-CCAGCCTCGTCCCGT AGA-3'. The PGPU6/GFP/Neo-St3ga15-mus-772 exhibited effective inhibition and was used in the subsequent investigation (GenePharma Co., Ltd., Shanghai, China).
Modulating ganglioside expression in the Hepal-6 cells. To decrease the expression of GM3 in the Hepa1-6 cell, the cells were transfected with the GM3 synthase shRNA plasmid at $37^{\circ} \mathrm{C}$ in a $5 \% \mathrm{CO}_{2}$ atmosphere. After $48 \mathrm{~h}$ incubation, the cells were harvested and the inhibition of ganglioside synthesis was monitored by high performance thin layer chromatography (HPTLC). For the GM3 synthase shRNA, the cells were transfected with PGPU6/GFP/Neo-St3ga15-mus-772 using lipofectamine ${ }^{\mathrm{TM}} 2000$ in opti-MEM medium. After $48 \mathrm{~h}$ transfection, the cells were harvested and the expression of GM3 synthase was monitored by RT-qPCR. To increase the expression of ganglioside in the Hepa1-6 cell, the cells were treated with $50 \mu \mathrm{M} \mathrm{GM} 3$ or $50 \mu \mathrm{M}$ GM2 for $48 \mathrm{~h}$ prior to harvesting.

Analysis of ganglioside expression in Hepal-6 cell by HPTLC. HPTLC analysis was performed, as described previously (18). Briefly, the cells were grown in $10 \mathrm{~cm}$ dishes until $\sim 90 \%$ confluent. The cells were then trypsinized and washed three times with phosphate-buffered saline (PBS; Sangon Biotech, Shanghai, China). The total lipids were extracted twice from the cell pellet using chloroform/methanol (1:1). The extracts were then combined and dried under a stream of $\mathrm{N}_{2}$. The gangliosides were purified by partitioning the dried total lipid in di-isopropyl ether/1-butanol/17 $\mathrm{mM}$ aqueous $\mathrm{NaCl}$ followed by sephadex G-50 gel filtration and lyophilization. The individual gangliosides were separated onto silica gel 60 HPTLC plates (Merck KGaA, Darmstadt) with a solvent system of chloroform/methanol $/ 0.25 \%$ aqueous $\mathrm{CaCl} 2 \cdot 2 \mathrm{H} 2 \mathrm{O}$ (60:40:9; Sangon Biotech). The gangliosides were visualized as purple bands by spraying them with resorcinol-HCI reagent and heating the plate at $120^{\circ} \mathrm{C}$.

In vitro cell migration assay. The cell migration assays were performed using a Boyden chamber (Corning Costar, Cambridge, MA, USA) with $8 \mu \mathrm{m}$ pore polycarbonate filters (BD Biosciences, Franklin Lakes, NJ, USA). The Hepa1-6 cells were treated with GM3 and transfected with the GM3 synthase shRNA plasmid, as described above. To inhibit the phosphatidylinositol 3-kinase (PI3K)/Akt pathway with LY294002, the Hepa1-6 cells treated, as above, were exposed to $15 \mu \mathrm{M} \mathrm{LY} 294002$ for $4 \mathrm{~h}$. The cells were then harvested and used for migration assays.

Following incubation of the cells in serum-free medium overnight, the cells $\left(1 \times 10^{5}\right)$ were resuspended in $300 \mu$ l serum-free medium containing $50 \mathrm{ng} / \mathrm{ml}$ EGF or $100 \mathrm{ng} / \mathrm{ml} \mathrm{HGF}$ and the desired agents and placed in the top compartment of the chamber. Subsequently, $250 \mu 1$ 10\% FBS medium was placed in the bottom chamber. After $36 \mathrm{~h}$ incubation at $37^{\circ} \mathrm{C}$ in a $5 \% \mathrm{CO}_{2}$ incubator, the cells on the top membrane surface were mechanically removed. The cells that migrated to the lower surface of the membrane were fixed and stained with $0.1 \%$ DAPI. Images of the cells were captured and the stained cells from five randomly selected fields were counted under a microscope (Nikon Corporation, Shanghai, China).

Western blot analysis. The cells treated, as described above, were seeded into 12-well plates (Falcon, BD Biosciences) and incubated overnight in serum-free medium containing the desired agents, as described in the figure legends. The medium was changed and the cells were stimulated with $50 \mathrm{ng} / \mathrm{ml}$ EGF 
A

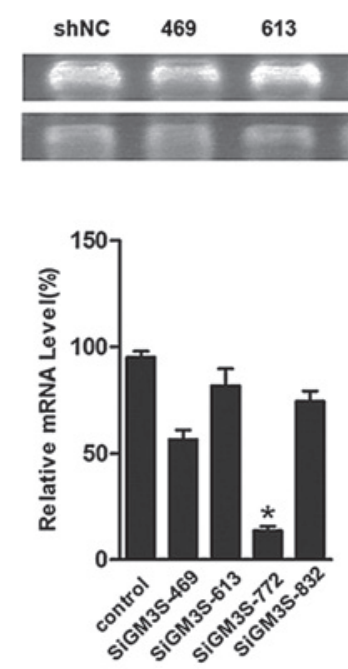

B

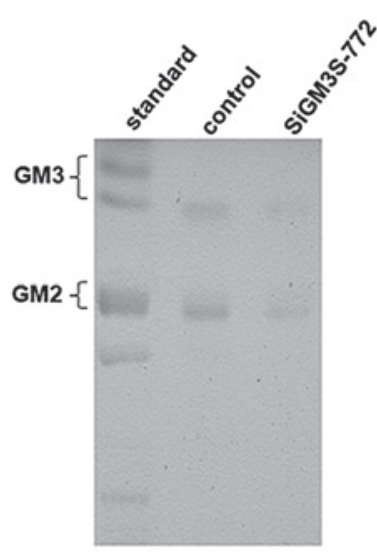

Gangliosides expressed in Hepa1-6

Figure 1. Suppression of the expression of GM3 synthase by transfection with SiGM3S plasmids. (A) Hepa1-6 cells were transfected with four different SiGM3S plasmids: PGPU6/GFP/Neo-St3ga15-mus-469, PGPU6/GFP/Neo-St3ga15-mus-613, PGPU6/GFP/Neo-St3ga15-mus-772, PGPU6/GFP/Neo-St3ga15-mus-832 and PGPU6/GFP/Neo-shNC (control). After $48 \mathrm{~h}$ transfection, the cells were harvested and the gene expression of GM3 synthase was analyzed by reverse transcription quantitative polymerase chain reaction. Data are expressed as the mean \pm standard deviation and were analyzed by one-way analysis of variance (Dunnett's test). ${ }^{*} \mathrm{P}<0.05$ between the different treatments. (B) Hepa1-6 cells were seeded into 12-well plates in RPMI-1640 medium supplemented with $10 \%$ fetal bovine serum and were transfected with the PGPU6/GFP/Neo-St3ga15-mus-772 SiGM3S. After $48 \mathrm{~h}$ incubation, the cells were harvested and the inhibition of GM3 synthesis was monitored by high performance thin layer chromatography. siGM3S, GM3 synthase shRNA; GFP, green fluorescent protein; shRNA, short hairpin RNA.

or $100 \mathrm{ng} / \mathrm{ml} \mathrm{HGF}$ for $10 \mathrm{~min}$ at room temperature or left unstimulated as a control. The cells were harvested and lysed in $200 \mu \mathrm{l}$ radioimmunoprecipitation assay buffer containing $1 \%$ Triton X-100, $150 \mathrm{mM} \mathrm{NaCl}, 25 \mathrm{mM}$ Tris ( $\mathrm{pH} 7.5$ ), $0.5 \%$ sodium deoxycholate, $0.1 \%$ SDS, $5 \mathrm{mM}$ pyrophosphate and $50 \mathrm{mM} \mathrm{NaF}$ with $1 \mathrm{mM} \mathrm{Na}_{3} \mathrm{VO}_{4}, 1 \mathrm{mM}$ DTT, $1 \%$ protease inhibitor cocktail and $1 \%$ phosphatase inhibitor cocktail per well. The lysate was subjected to SDS-PAGE using $10 \%$ gel. Following electrophoresis, the proteins were transferred onto polyvinylidene difluoride (PVDF) membranes at $100 \mathrm{~mA}$ for $2 \mathrm{~h}$. The non-specific binding sites on the PVDF membranes were inhibited using 3\% (w/v) bovine serum albumin. The target protein bands in the PVDF membranes were revealed by immunoblotting with the primary antibodies given in the 'antibodies' section of 'Materials and methods' and detected using enhanced chemiluminescence reagents (Amersham Pharmacia Biotech, Amersham, UK).

\section{Results}

Suppression of GM3 synthesis by RNAi targeting GM3 synthase in Hepal- 6 cells. To decrease the expression of GM3 in the Hepa1-6 cells, the cells were transfected with GM3 synthase shRNA plasmids. The RT-qPCR results revealed that, among the four GM3 synthase shRNA plasmids, the PGPU6/GFP/Neo-St3ga15-mus-772 plasmid was the most effective at inhibiting the expression of GM3 synthase (Fig. 1A). The inhibitory effect was monitored by HPTLC (Fig. 1B).

Effect of GM3 on in vitro motility and migration of Hepal-6 cell stimulated with EGF and HGF. The effects of GM3 on the motility and migration of the EGF- or HGF-stimulated Hepa1-6 cells were investigated. A decrease in GM3 content by the GM3 synthase shRNA plasmid promoted EGF-stimulated cell migration (Fig. 2a and b) and inhibited HGF-stimulated migration (Fig. 2e and f). Conversely, an increase in GM3 content, caused by the addition of exogenous GM3 in the culture medium, inhibited EGF-stimulated cell migration (Fig. 2a and c) and promoted HGF-stimulated migration (Fig. 2e and g). The addition of exogenous GM2 had no effect on the migration of the Hepa1-6cells treated with EGF and HGF (Fig. 2a, d and e, h). These results indicated that GM3, but not GM2, inhibited EGF-stimulated cell migration and promoted the HGF-stimulated migration in vitro.

Effect of GM3 on the phosphorylation of EGFR and HGFR. To elucidate the precise molecular mechanism by which GM3 affected EGF- and HGF-stimulated migration of the Hepa1-6 cells, the present study examined the effect of GM3 on the phosphorylation of EGFR and HGFR. Under control conditions, no background phosphorylation at either Tyr-1173 or Tyr-845 was observed. However, a decrease in GM3 content, by treating cells with the GM3 synthase shRNA plasmid, significantly elevated the EGF-stimulated phosphorylation of EGFR at Tyr-1173 and reduced the EGF-stimulated phosphorylation of Tyr-845 (Fig. 3Aa). An increase in GM3 content, by treating cells with exogenous GM3, markedly inhibited the EGF-stimulated phosphorylation of EGFR at the Tyr-1173 and elevated the phosphorylation of Tyr- 845 (Fig. 3Ab). The phosphorylation of EGFR at Tyr-1068 and Tyr-1086 were not detected (data not shown). The results 

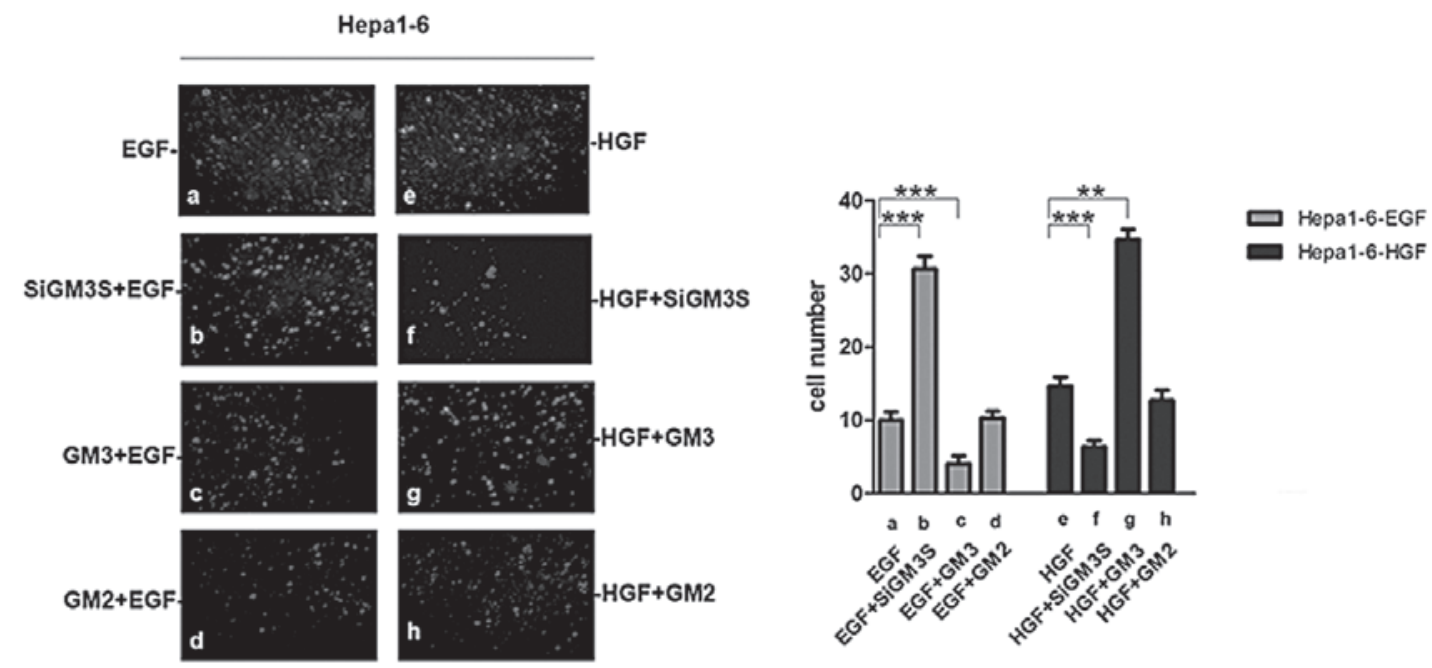

Figure 2. Effect of GM3 on the EGF- and HGF-induced motility and migration of Hepa1-6 cells in vitro. The Hepa1-6 cells were treated with $50 \mu$ M GM3 or $50 \mu \mathrm{M} \mathrm{GM} 2$ and transfected with the SiGM3S plasmid for $48 \mathrm{~h}$. In vitro cell migration assays were then performed. Data are expressed as the mean \pm standard deviation and were analyzed by a one-way analysis of variance (Dunnetts test). ${ }^{* *} \mathrm{P}<0.01$ and ${ }^{* * * *} \mathrm{P}<0.001$ between the different treatments. EGF, epidermal growth factor; HGF, hepatocyte growth factor; SiGM3S, GM3 synthase shRNA.

demonstrated that GM3 suppressed the EGF-stimulated phosphorylation of EGFR at Tyr-1173, but promoted the phosphorylation of Tyr-845. Tyr-1173 is located at the c-terminus of EGFR and is a major EGFR autophosphorylation site. Phosphorylated Tyr-1173 serves as a docking site for SH2-domain containing signaling molecules and leads to the activation of the downstream signaling pathways. Tyr- 845 is distinct from others located at the EGFR c-terminus. It is located in the kinase domain of the EGF receptor and can be transphosphorylated by src kinase (19). The significance of Tyr-845 phosphorylation remains to be elucidated, however, it may be involved in the modulation of tyrosine kinase activity of the receptor. These results suggested that the mechanism for the GM3-suppressed phosphorylation of EGFR in Hepa1-6 cells is complicated.

By contrast, a decrease in GM3 content, by treating cells with a GM3 synthase shRNA plasmid, significantly reduced the HGF-stimulated phosphorylation at Tyr-1313 and Tyr-1365 (Fig. 3Ba). An increase in GM3 content, by treating cells with exogenous GM3, markedly promoted the HGF-stimulated phosphorylation of cMet at Tyr-1313 and Tyr-1365 (Fig. 3Bb). The phosphorylation of Tyr-1349 was not detected (data not shown). GM3 promoted the HGF-stimulated phosphorylation of HGFR at Tyr-1313 and Tyr-1365.

These results indicated that GM3 suppressed the EGF-stimulated phosphorylation and activation of EGFR, but promoted the HGF-stimulated phosphorylation and activation of cMet, which may explain why GM3 exerted opposing affects on the motility and migration of the EGF- and HGF-stimulated Hepa1-6 cells.

Effect of GM3 on the activity of signaling pathways in Hepal-6 cells. It is well known that the autophosphorylation of tyrosine kinase receptors is an essential step for their activation, which results in phosphorylation at specific tyrosine residues on the intracellular domain of the receptors. Certain cytoplasmic SH2-domain containing signaling molecules, including PI3K,
PLC- $\gamma$, growth factor receptor-bound protein 2 and protein tyrosine phosphatases, bind to specific phosphotyrosine sequences on the receptor, thereby initiating the intracellular corresponding signaling pathways (20). To further investigate the mechanism underlying the different effects of GM3 on EGFand HGF-stimulated motility and migration in vitro, the present study investigated the effects of GM3 on the activity of intracellular signaling pathways, which are essential for the modulation of tumor cell invasion and migration. Three of the main downstream signal transduction pathways activated by GFRs are the MAPK, PLC $\gamma$ and PI3K/Akt pathways. The present study examined the effect of GM3 on the activity of these signaling pathways. Fig. 4 shows that an alteration in GM3 content had no significant affect on the phosphorylation of MAPK (p44/42) in the Hepa1-6 cells when stimulated by either EGF (Fig. 4Aa and b) or HGF (Fig. 4Ba and b). This result suggested that GM3 did not affect the activity of MAPK signaling in the Hepa1-6 cells. The effect of GM3 on the activity of the PLC $\gamma$ signaling pathway was also assessed. It is understood that the phosphorylation and translocation of PLC $\gamma$ from the cytosol to the plasma membrane are necessary for its activation and function (13). The present study attempted to examine the effect of GM3 on the phosphorylation of PLC $\gamma 1$, however phosphorylated PLC $\gamma 1$ was undetected in the control cells and in the cells treated with EGF or HGF (data not shown). The localization of PLC $\gamma 1$ in the cells was further examined, however, membrane-binding PLC $\gamma 1$ was not detected in the cells stimulated with either EGF or HGF (data not shown). As shown in Fig. 4, GM3 promoted the expression of PLC $\gamma 1$ in the cells stimulated with EGF (Fig. 4Ac and d) and inhibited the expression of PLC $\gamma 1$ in the cells stimulated with HGF (Fig. 4Bc and d). These data indicated that GM3 did not affect the activity of the PLC $\gamma 1$ signaling pathway. The phosphorylation of Akt was also examined. In the EGF-treated cells, suppression of GM3 synthesis by treatment of the cells with a GM3 synthase shRNA plasmid, elevated the phosphorylation of Akt at Ser 473 (Fig. 4Ae), whereas increasing the GM3 content by treating the cells with exogenous GM3 inhibited the 
A a

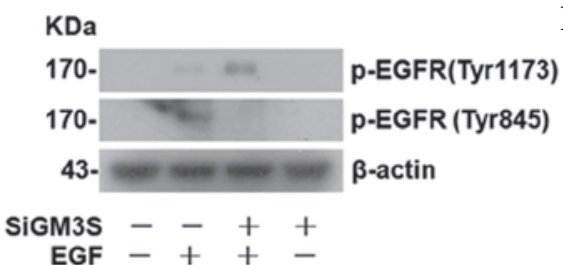

$\mathbf{b}$

$\mathrm{KDa}$

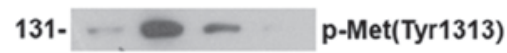

131- $\quad-m-M e t(T y r 1365)$

43- $\beta$-actin

SigM3S $-\quad++$

HGF -++-
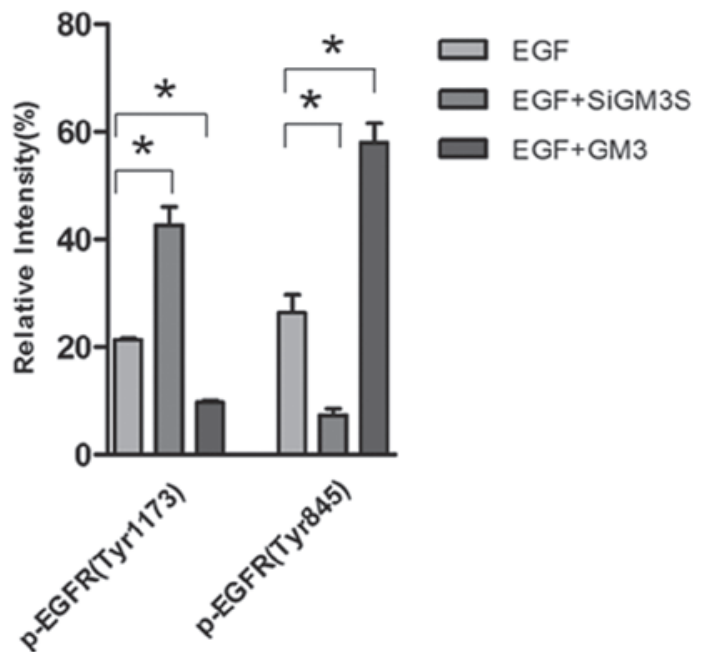

B

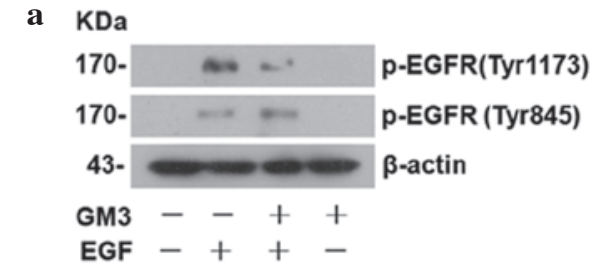

b
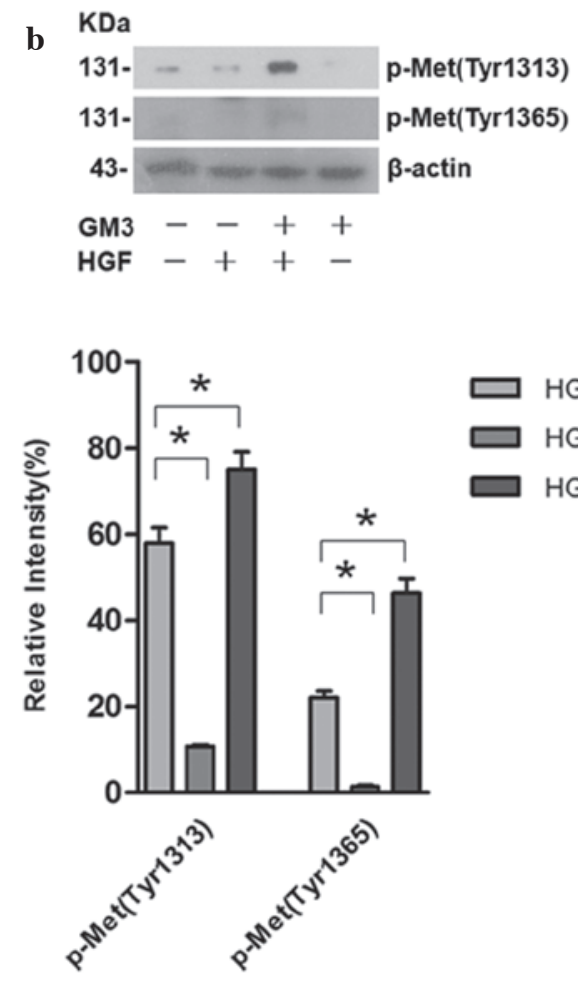

Figure 3. Effect of GM3 on the EGF-dependent autophosphorylation of EGFR and the HGF-dependent autophosphorylation of Met in the Hepa1-6 cells. (Aa) Decrease in GM3 content induced by treating cells with a SiGM3S shRNA plasmid significantly elevated the EGF-stimulated phosphorylation of EGFR at Tyr-1173 and reduced EGF-stimulated phosphorylation at Tyr-845. (Ab) An increase in GM3 content induced by treating cells with exogenous GM3 markedly inhibited EGF-stimulated phosphorylation of EGFR at the Tyr-1173 and elevated the phosphorylation at Tyr-845 . (Ba) Decrease in GM3 content induced by treating cells with a SiGM3S shRNA plasmid significantly reduced the HGF-stimulated phosphorylation at Tyr-1313 and Tyr-1365 (Bb) An increase in GM3 content induced by treating cells with exogenous GM3 markedly increased HGF-stimulated phosphorylation of cMet at Tyr-1313 and Tyr-1365. Treated Hepa1-6 cells were serum-starved for $12 \mathrm{~h}$ in the presence of the desired reagents and then stimulated with $50 \mathrm{ng} / \mathrm{ml} \mathrm{EGF} \mathrm{or} 100 \mathrm{ng} / \mathrm{ml} \mathrm{HGF}$ for $10 \mathrm{~min}$. The cells were then harvested and subjected to western blot analysis with antibodies, as described in the Materials and methods. All experiments were performed at least three times. Data are expressed as the mean \pm standard deviation and were analyzed by a one-way analysis of variance (Dunnett's test). ${ }^{*} \mathrm{P}<0.05$ between the different treatments. EGF, epidermal growth factor; EGFR, EGF receptor; HGF, hepatocyte growth factor; Met, HGF receptor; p-, phosphorylated; SiGM3S, GM3 synthase shRNA plasmid.

phosphorylation of Akt at Ser 473 (Fig. 4Af). By contrast, in the HGF-treated cells, suppression of GM3 synthesis by treatment of the cells with a GM3 synthase shRNA plasmid inhibited the phosphorylation of Akt at Ser 473 (Fig. 4Be). An increase in the GM3 content following treatment with exogenous GM3 promoted the phosphorylation of Akt at Ser 473 (Fig. 4Bf). These results suggested that GM3 suppressed the EGF-stimulated activation of the PI3K/Akt signaling pathway and promoted the HGF-stimulated activation of the PI3K/Akt signaling pathway, indicating that the PI3K/Akt signaling pathway may be important in GM3-regulated cell migration.

Effect of inhibiting the PI3K/Akt pathway on cell mobility and migration. To confirm whether Hepa1- 6 cell motility and migration were mainly regulated by the PI3K/Akt signaling pathway, the activity of PI3K in cells was inhib- ited using LY294002, an inhibitor of PI3K. Inhibiting the PI3K/Akt pathway with LY294002 reversed the positive effect following transfection with a GM3 synthase shRNA plasmid (Fig. 5a-c) on EGF-stimulated migration. The positive effect on HGF-stimulated migration, caused by the addition of exogenous GM3, was also reduced by inhibiting the PI3K/Akt pathway (Fig. 5d-f). This finding confirmed that the PI3K/Akt pathway was involved in the modulation of Hepa1- 6 cell motility and migration in vitro.

\section{Discussion}

Several studies have demonstrated that expression of the ganglioside GM3 is associated with the malignant behavior of cancer cells, including cell proliferation, adhesion, invasion and metastasis (21). However, the precise mechanism 
A

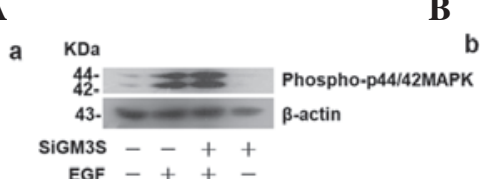

EGF - + + -

C KDa

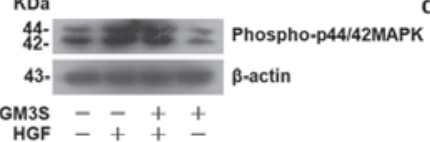

e KDa

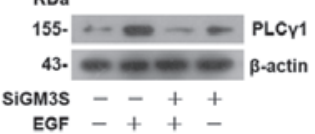

EGF - + +

g

$\mathrm{KDa}$

155- $+\cdots$ PLCY1

43- $=-2=-\beta$-actin

SIGM3S - - + +

HGF -++

i $\mathrm{KDa}$

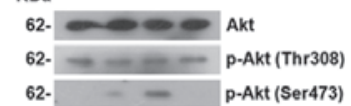

62- - p-Akt (Ser473)

43. $-3-a=0-a c t i n$

SiGM3S - - +

EGF - + +

k KDa

62. $-=-2=$ Akt

62. - - - - p-Akt (Thr308)

62. - D-Akt (Ser473)

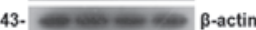

SiGM3S --++
HGF -+++

B b KDa

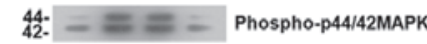

43. $=0$-actin

GM3 $-\overline{+}+$

d KDa

42. $838 \equiv$ Phospho-p44/42MAPK

43- $\beta$-actin

GM3 $-\overline{+}+$

f KDa

155. - - - PLCY1

43- $\beta$-actin

GM3 - -++

EGF -++-

h

KDa

155. - - PLCY1

43- $-=-\infty$-actin

GM3 - - +

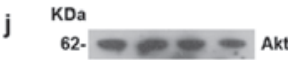

62- $-5-0$ p-Akt (Thr308)

62- - p-Akt (Ser473)

43. $\beta$-actin

GM3 - - +

KDa

62- $-2=-$ Akt

62- - - p-Akt (Thr308)

62. $\quad-$ p-Akt (Ser473)

43. $=--\beta$-actin

GM3 $-\overline{+}+$
HGF -++
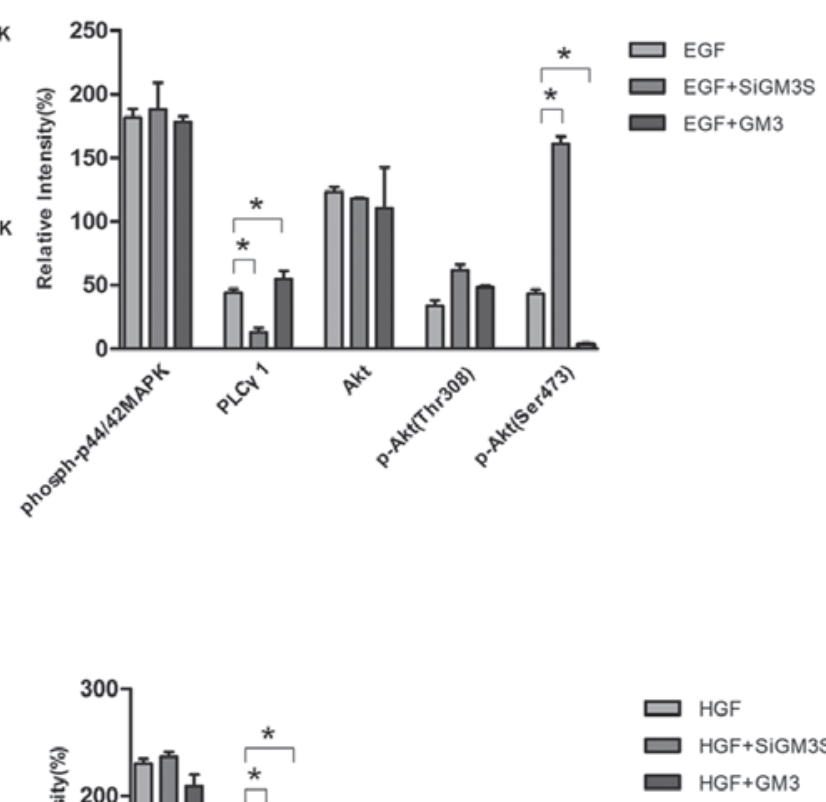

Figure 4. (Aa, c, e, g, i, k and Bb, d, f, h, j, l) Effect of GM3 on the EGF- and HGF-dependent signaling pathways in the Hepa1-6 cells. The treated Hepa1-6 cells were serum-starved for $12 \mathrm{~h}$ in the presence of the desired reagents and then stimulated with $50 \mathrm{ng} / \mathrm{ml} \mathrm{EGF}$ or $100 \mathrm{ng} / \mathrm{ml} \mathrm{HGF}$ for $10 \mathrm{~min}$. The cells were then harvested and subjected to western blot analysis with antibodies, as described in the Materials and methods. All experiments were performed at least three times. Data are expressed as the mean \pm standard deviation and were analyzed by one-way analysis of variance (Dunnett's test). ${ }^{*} \mathrm{P}<0.05$ between the different treatments. EGF, epidermal growth factor; HGF, hepatocyte growth factor; PLC $\gamma 1$, phospholipase C $\gamma 1$; MAPK, mitogen-activated protein kinase; p-, phosphorylated; SiGM3S, GM3 synthase shRNA plasmid.

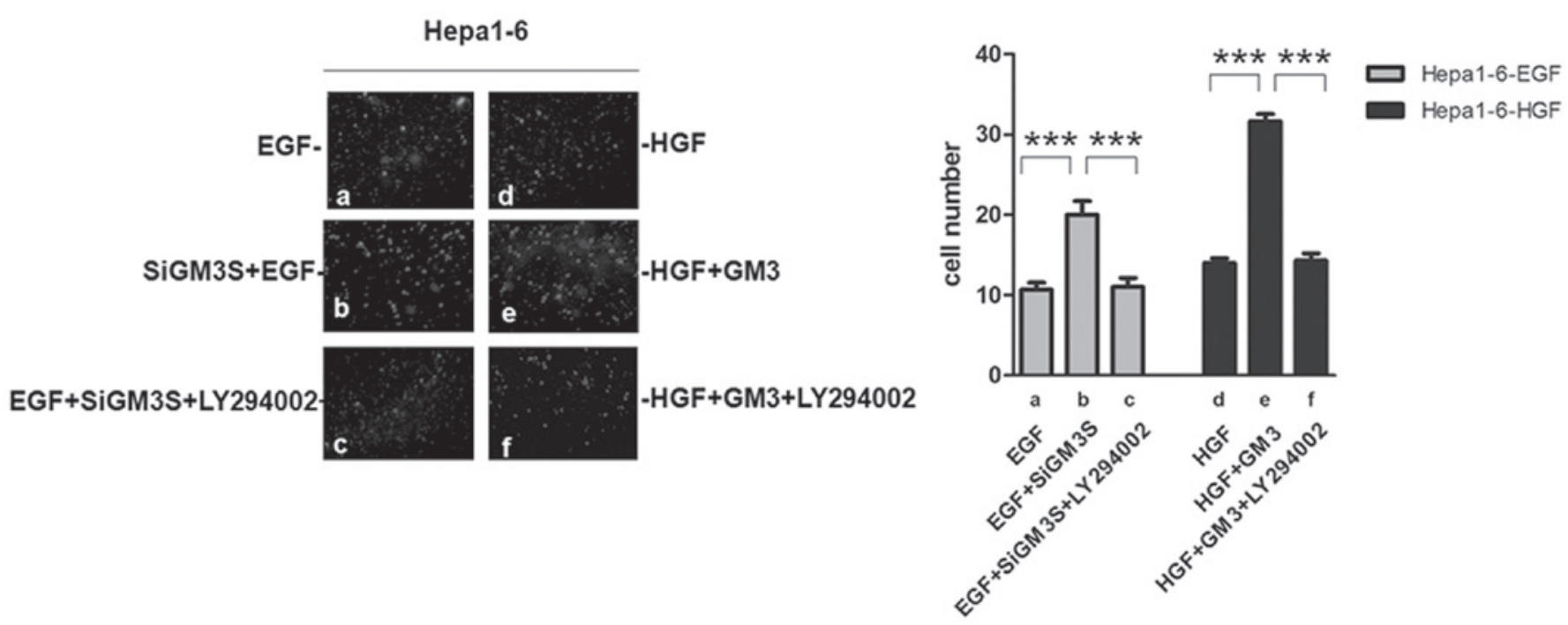

Figure 5. Effect of inhibiting the PI3K/Akt pathway on the mobility and migration of Hepa1-6 cells in vitro. Following treatment of the Hepa1-6 cells with GM3 or SiGM3S for $48 \mathrm{~h}$, the PI3K/Akt signaling pathway in the Hepa1-6 cells was inhibited with $15 \mu \mathrm{M}$ LY294002 for 4 h and the cells were then subjected to a migration assay, as described in the Materials and methods. Data are expressed as the mean \pm standard deviation and were analyzed by a one-way analysis of variance (Dunnett's test). ${ }^{* * *} \mathrm{P}<0.001$ between the different treatment groups. EGF, epidermal growth factor; HGF, hepatocyte growth factor; SiGM3S, GM3 synthase shRNA plasmid; PI3K, phosphoinositide 3-kinase. 
of this effect remains to be elucidated. One major problem is that GM3 exerts different, even opposite, actions on different types of tumor. Several mechanisms that underline the actions of GM3 have been reported. GM3 interacts with basal membrane components, including adhesion molecules, to regulate cell adhesion and migration and GM3 directly interacts with GFRs, including EGFR, fibroblast growth factor receptor, platelet-derived growth factor receptor, vascular endothelial growth factor and insulin receptor, to modulate the receptor function and subsequently affect the intracellular signaling pathways (20-23). GM3 also indirectly regulates GFR activity by affecting the interaction between GFR and membrane proteins, including integrins, CD9 and CD82 tetraspanins (24-26) or by affecting the intracellular Src kinase and protein tyrosine phosphatase activity (27-29). GM3 also regulates the activation of non-tyrosine kinase receptors, including G-protein coupled receptors (30). Thus, the mechanisms by which GM3 function are particularly complicated. In different types of tumor or under different physiological or pathological conditions, the expression levels of molecules associated with GM3, including growth factors, adhesion molecules and other membrane lipids differ and, consequently, GM3 exerts its actions by different mechanisms (31).

As EGF and $\mathrm{HGF}$ are important cytokines regulating various cell processes, including cell proliferation, differentiation, apoptosis and oncogenesis, Hepa1-6 cells contain mainly GM3. In the present study, GM2 had no effect on the migration of Hepa1-6 cells treated with EGF and HGF. GM3 suppressed the EGF-stimulated autophosphorylation of EGFR at tyr1173 and subsequently downregulated the PI3K/Akt signaling pathway. Conversely, GM3 enhanced the HGF-stimulated autophosphorylation of HGFR at Tyr-1313 and Tyr-1365 and subsequently upregulated the PI3K/Akt signaling pathway. This finding may, at least in part, explain why GM3 had opposing effects on the EGF- and HGF-induced Hepa1-6 cell motility and migration in vitro and may explain why GM3 exerts its actions in a tissue or tumor-type specific manner.

\section{Acknowledgements}

This study was support by grants from the National Natural Science Foundation of China (grant no. 81401738) and Liaoning Province Science and Technology Project (grant no. 2013023041).

\section{References}

1. Huwiler A, Kolter T, Pfeilschifter J and Sandhoff K: Physiology and pathophysiology of sphingolipid metabolism and signaling. Biochim Biophys Acta 1485: 63-99, 2000.

2. Toledo MS, Suzuki E, Handa K and Hakomori S: Cell growth regulation through GM3-enriched microdomain (glycosynapse) in human lung embryonal fibroblast WI38 and its oncogenic transformant VA13. J Biol Chem 279: 34655-34664, 2004 .

3. Hakomori S: Glycosphingolipids in cellular interaction, differentiation and oncogenesis. Annu Rev Biochem 50: 733-764, 1981.

4. Regina-Todeschini A and Hakomori SI: Functional role of glycosphingolipids and gangliosides in control of cell adhesion, motility, and growth, through glycosynaptic microdomains. Biochim Biophys Acta 1780: 421-433, 2008.
5. Hakomori SI: Glycosynaptic microdomains controlling tumor cell phenotype through alteration of cell growth, adhesion, and motility. FEBS Lett 584: 1901-1906, 2010.

6. Van Echten G and Sandhoff K: Ganglioside metabolism. Enzymology, topology, and regulation. J Biol Chem 268: 5341-5344, 1993

7. Iwabuchi K, Yamamura S, Prinetti A, Handa K and Hakomori S: GM3 - enriched microdomain involvedin cell adhesion and signal transduction through carbohydrate-carbohydrate interaction in mouse melanoma B16 cells. J Biol Chem 273: 9130-9138, 1998

8. Kojima $\mathrm{N}$ and Hakomori S: Cell adhesion, spreading, and motility of GM3-expressing cells based on glycolipid-glycolipid interaction. J Biol Chem 266: 17552-17558, 1991.

9. Hakomori S: Tumor malignancy defined by aberrant glycosylation and sphingo (glyco) lipid metabolism. Cancer Res 56: 5309-5318, 1996.

10. Birklé S, Zeng G, Gao L, Yu RK and Aubry J: Role of tumor-associated gangliosides in cancer progression. Biochimie 85: 455-463, 2003.

11. Fujimoto Y, Izumoto S, Suzuki T, et al: Ganglioside GM3 inhibits proliferation and invasion of glioma. Neurooncol 71: 99-106, 2005.

12. Kawamura S, Ohyama C, Watanabe R, et al: Glycolipid composition in bladder tumor: a crucial role of GM3 ganglioside in tumor invasion. Int J Cancer 94: 343-347, 2001.

13. Wang XQ, Sun P and Paller AS: Ganglioside GM3 blocks the activation of epidermal growth factor receptor induced by integrin at specific tyrosine sites. J Biol Chem 278: 48770-48778, 2003.

14. Wang XQ, Sun P, Go L, et al: Ganglioside GM3 promotes carcinoma cell proliferation via urokinase plasminogen activator-induced extracellular signal-regulated kinase-independent p70S6 kinase signaling. J Invest Dermatol 6: 2687-2696, 2006.

15. Mitsuzuka K, Handa K, Satoh M, Arai Y and Hakomori S: A specific microdomain ("glycosynapse 3") controls phenotypic conversion and reversion of bladder cancer cells through GM3-mediated interaction of alpha3beta1 integrin with CD9. J Biol Chem 280: 35545-35553, 2005.

16. Saha S and Mohanty KC: Enhancement of metastatic potential of mouse B16-melanoma cells to lung after treatment with gangliosides of B-16-melanoma cells of higher metastatic potential to lung. Indian J Exp Biol 41: 1253-1258, 2003.

17. Gu Y,Zhang J, Mi W, et al: Silencing of GM3 synthase suppresses lung metastasis of murine breast cancer cells. Breast Cancer Res 10: 1-12, 2008.

18. Ladisch S and Gillard B: A solvent partition method for microscale ganglioside purification. Anal Biochem 146: 220-231, 1985.

19. Biscardi JS, Maa MC, Tice DA, et al: c-Src-mediated phosphorylation of the epidermal growth factor receptor on Tyr845 and Tyr1101 is associated with modulation of receptor function. J Biol Chem 274: 8335-8343, 1999.

20. Bremer EG and Hakomori S: GM3 ganglioside induces hamster fibroblast growth factor inhibition in chemically defined medium: ganglioside may regulate growth factor receptor function. Biochem Biophys Res Commun 106: 711-728, 1982.

21. Rebbaa A, Hurh J, Yamamoto K, Kersey D and Bremer EG: Ganglioside GM3 inhibition of EGF receptor mediated signal transduction. Glycobiology 6: 399-406, 1996.

22. Meuillet E, Cremel G, Dreyfus H and Hicks D: Differential modulation of basic fibroblast and epidermal growth factor receptor activation by ganglioside GM3 in cultured retinal Muller glia. Glia 17: 206-216, 1996.

23. Yates AJ, Saqr HE and Van Brocklyn J: Ganglioside modulation of PDGF receptor. A model for ganglioside functions. J Neurooncology 24: 65-73, 1995.

24. Mukherjee P, Faber AC, Shelton LM, et al: Thematic review series: sphingolipids. Ganglioside GM3 suppresses the proangiogenic effects of vascular endothelial growth factor and ganglioside GD1a. J Lipid Res 49: 929-938, 2008.

25. Chung TW, Kim SJ, Choi HJ, et al: Ganglioside GM3 inhibits VEGF/VEGFR-2-mediated angiogenesis: direct interaction of GM3 with VEGFR-2. Glycobiology 19: 229-239, 2009.

26. Kawakami Y, Kawakami K, Steelant WF, et al: Tetraspanin CD9 is a "proteolipid," and its interaction with alpha 3 integrin in microdomain is promoted by GM3 ganglioside, leading to inhibition of laminin-5-dependent cell motility. J Biol Chem 277: 34349-34358, 2002. 
27. Ono M, Handa K, Sonnino S, et al: GM3 ganglioside inhibits CD9-facilitated haptotactic cell motility: coexpression of GM3 and CD9 is essential in the downregulation of tumor cell motility and malignancy. Biochemistry 40: 6414-6421, 2001.

28. Todeschini AR, Dos Santos JN, Handa K and Hakomori SI: Ganglioside GM2/GM3 complex affixed on silica nanospheres strongly inhibits cell motility through CD82/cMet-mediated pathway. Proc Natl Acad Sci USA 105: 1925-1930, 2008

29. Suárez Pestana E, Greiser U, Sánchez B, et al: Growth inhibition of human lung adenocarcinoma cells by antibodies against epidermal growth factor receptor and by ganglioside GM3 involvement of receptor-directed protein tyrosine phosphatase(s). Br J Cancer 75: 213-220, 1997.
30. Suárez Pestana E, Tenev T, Gross S, et al: The transmembrane protein tyrosine phosphatase RPTP sigma modulates signaling of the epidermal growth factor receptor in A431 cells. Oncogene 18: 4069-4079, 1999.

31. Bassi R, Viani P, Giussani P, Riboni L and Tettamanti G: GM3ganglioside inhibits endothelin-1-mediated signal transduction in C6 glioma cells. FEBS Lett 507: 101-104, 2001. 